\title{
Morphology of perforated atrial septal aneurysm suitable for closure by transcatheter device placement
}

\author{
P Ewert, F Berger, M Vogel, I Dähnert, V Alexi-Meshkishvili, P E Lange
}

\begin{abstract}
Objective-To define the morphological criteria of perforated atrial septal aneurysms suitable for closure by a transcatheter device.

Methods-A retrospective analysis of all consecutive patients with atrial septal aneurysm and one or more perforations presenting between May 1997 and June 1999. The aneurysms were classified as: aneurysm with persistent foramen ovale (type A); aneurysm with single atrial septal defect (type B); aneurysm with two perforations requiring more than one device for closure (type C); and aneurysm with multiple perforations (type D).

Patients-Data from 50 patients aged 5-78 years (mean 43 years) were analysed; 32 had systemic thromboembolism or transient ischaemic attacks, eight presented with dyspnoea on exercise, and 10 were discovered incidentally but had significant left to right shunt and right ventricular volume overload.

Results-In all 18 patients with aneurysm and persistent foramen ovale (type A), transcatheter closure was possible. In nine with aneurysm and atrial septal defect (type B), five defects were closed and four required surgery. Device closure was achieved in all 10 patients with aneurysms and two perforations (type C), but four had a residual shunt. Thirteen patients with multiple perforated aneurysms (type D) underwent surgery.
\end{abstract}

Conclusions-This classification of morphology of perforations of aneurysm is clinically useful for selecting patients for treatment by transcatheter devices.

(Heart 2000;84:327-331)

Keywords: aneurysm; atrial septum; transcatheter device

The prevalence of atrial septal aneurysm is reported to be $2.2 \% .^{1}$ Perforated aneurysms may be associated with a significant left to right shunt and present like an atrial septal defect, ${ }^{2}$ or the shunt may be small and clinically present as systemic thromboembolic events caused either by thrombus formation inside the aneurysm or by paradoxical embolism. ${ }^{3}$ In some patients devoid of clinical symptoms, the aneurysm may be detected incidentally on medical screening. ${ }^{4}$ There are no universally accepted treatment strategies, and recommendationswhich vary from country to country-include anticoagulation $^{5}$ or closure, depending on the clinical symptoms, the morphology of the aneurysm, and the presence and number of perforations. ${ }^{6}$ Recent improvements in transcatheter devices for closing atrial septal defects have led to attempts to use this technique for the treatment of perforated aneurysms. ${ }^{78}$ Our aim in this study was to analyse the morphology of perforated atrial septal aneurysms to determine which of them might be suitable for closure by transcatheter device placement.

\section{Methods}

\section{PATIENT SELECTION}

Consecutive patients with a perforated atrial septal aneurysm as an isolated congenital malformation of the heart and who presented between May 1997 and June 1999 at our institution were included in this retrospective study. The start date represents the day of the first implantation of an Amplatzer septal occluder at our centre. ${ }^{9}$ Atrial septal aneurysm was considered to be present ${ }^{10}$ if there was circumscribed bulging in the atrial septum, with a base width of at least $15 \mathrm{~mm}$ (or $8 \mathrm{~mm} / \mathrm{m}^{2}$ body surface area in children) and a depth of at least $15 \mathrm{~mm}$ (or $8 \mathrm{~mm} / \mathrm{m}^{2}$ body surface area in children), or if there was an oscillating membrane with excursions of $15 \mathrm{~mm}$ or more. In all patients, diagnosis was made by transthoracic echocardiography using commercially available sector scanners (Vingmed System V, Horten, Norway). All patients also underwent an evaluation by transoesophageal echocardiography (TOE). Fifty patients were identified. In 22 of these, a history of previous thromboembolic events with cerebral infarction had been documented by cranial computed or magnetic resonance tomography, and a further 10 had recurrent transient ischaemic attacks. Eight presented with dyspnoea on exercise. The remaining 10 patients were discovered incidentally on medical screening, and had echocardiographic signs of right ventricular volume overload. Two patients had atrial flutter on presentation, while the remainder were in sinus rhythm.

\section{CARDIAC CATHETERISATION}

All patients underwent cardiac catheterisation on the basis that the perforated atrial septal aneurysm would be treated with a transcatheter device. Standard fluid filled catheters were employed, and the pulmonary $(\mathrm{Qp})$ to systemic 


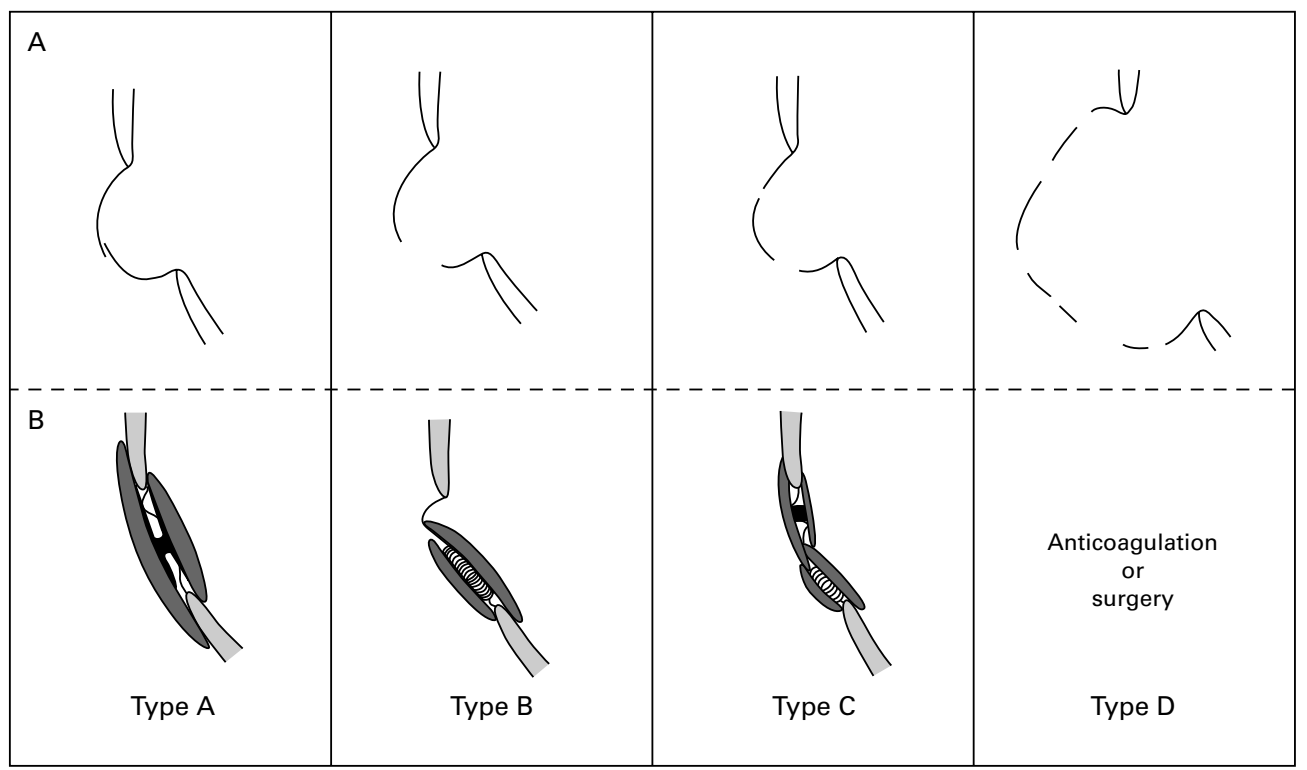

Figure 1 Classification of perforated atrial septal aneurysms with respect to the morphology of the interatrial communications $(A)$ and their possible treatment (B). Type A: persistent foramen ovale (PFA), occluded with a large PFA occluder. Type B: atrial septal defect (ASD), occluded with an Amplatzer ASD occluder. Type C: two or few perforations located in no more than two clusters and not treatable with a single device, treated with an Amplatzer PFA occluder and an Amplatzer ASD occluder. Type D: multiple perforations spread irregularly over the aneurysm-transcatheter treatment is currently not recommended.

(Qs) flow ratio was measured by oximetry using the Fick principle. The size of the interatrial communications was determined with balloon catheters, as described elsewhere. ${ }^{911}$ All patients had TOE as part of the routine protocol during balloon sizing, which allowed the detection and localisation of additional defects by colour flow mapping during balloon occlusion. ${ }^{9}$ On the basis of the findings at TOE, the aneurysms were classified as follows (fig 1A):

- atrial septal aneurysm with patent foramen ovale (type A);

- atrial septal aneurysm with single atrial septal defect (type B);

- atrial septal aneurysm with two perforations or few perforations located in not more than two clusters requiring placement of more than one device (type C); and

- atrial septal aneurysm with multiple perforations located in more than two areas of the atrial septum (type D).

Closure of interatrial communications by transcatheter device placement was attempted in all patients. We used three devices: the Amplatzer septal (ASD) occluder, the Amplatzer persistent foramen ovale (PFA) occluder, and the Cardio-Seal device. The technical details of the implantation of the Cardio-Seal (Nitinol Medical Technologies, Boston, Massachusetts, USA) and Amplatzer devices (AGA Medical Corporation, Golden Valley, Minnesota, USA) have been described elsewhere. ${ }^{8}{ }^{12}$ A $7-14$ French long sheath was used to deliver the Amplatzer or Cardio-Seal devices. TOE was performed in all patients using a multiplane transoesophageal probe interfaced with a Vingmed 800 sector scanner or a monoplane probe interfaced with a Siemens Sonoline 1200 sector scanner (Siemens, Erlangen, Germany) to monitor device placement. All patients were sedated with intravenous midazolam $0.1-0.2 \mathrm{mg} / \mathrm{kg}$ immediately before entering the catheterisation laboratory. During the procedure, sedation was achieved by a continuous infusion of propofol 3-5 mg/kg/h. Neither endotracheal intubation nor mechanical ventilation was required in any patient. All patients in whom transcatheter device closure of defects was achieved were treated with aspirin $3-5 \mathrm{mg} / \mathrm{kg} /$ day for six months.

All the patients, or their parents, gave written informed consent for defect closure with either the Amplatzer or the Cardio-Seal device. The implantation protocol was approved by the local institutional ethics committee.

\section{Results}

Data are expressed as mean (SD) or median (range). The 50 patients were treated at a mean age of 43 (17) years (range 5-78 years); four were under 18 years of age. In the 18 who presented without stroke but with signs of right ventricular volume overload, the mean (SD) pulmonary $(\mathrm{Qp})$ to systemic (Qs) flow ratio was $2.1(0.6)$. In the 18 patients with atrial septal aneurysm and persistent foramen ovale (type A), transcatheter closure was possible in all (figs 2 and 3) using the Amplatzer PFO occluder ( $\mathrm{n}=9$, fig $1 \mathrm{~B})$, the Amplatzer ASD occluder $(n=8)$, or the Cardio-Seal device $(\mathrm{n}=1)$.

In the nine patients with atrial septal aneurysm and atrial septal defect (type B), an Amplatzer ASD occluder was placed in five (figs $1 \mathrm{~B}$ and 2 ). In the remaining four patients, the defects were located too close to the mitral valve in two patients and were too large (> $28 \mathrm{~mm}$ and $>34 \mathrm{~mm}$ diameter) for closure with the available devices at that time in the other two. These patients underwent surgery. In the five who were successfully treated with 


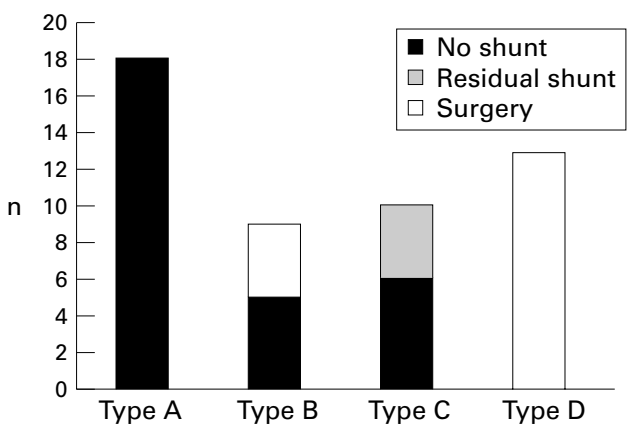

Figure 2 Results of the treatment of 50 perforated atrial septal aneurysms. Black bars show successful interventional closure and grey bars interventional closure with residual shunts. White bars indicate no intervention except surgical closure.

an Amplatzer ASD occluder, a small part of the atrial septal aneurysm remained uncovered by the device, but was stabilised by the splinting effect of the occluder.

In the 10 patients with atrial septal aneurysm and two perforations, or perforations located in not more than two clusters that required
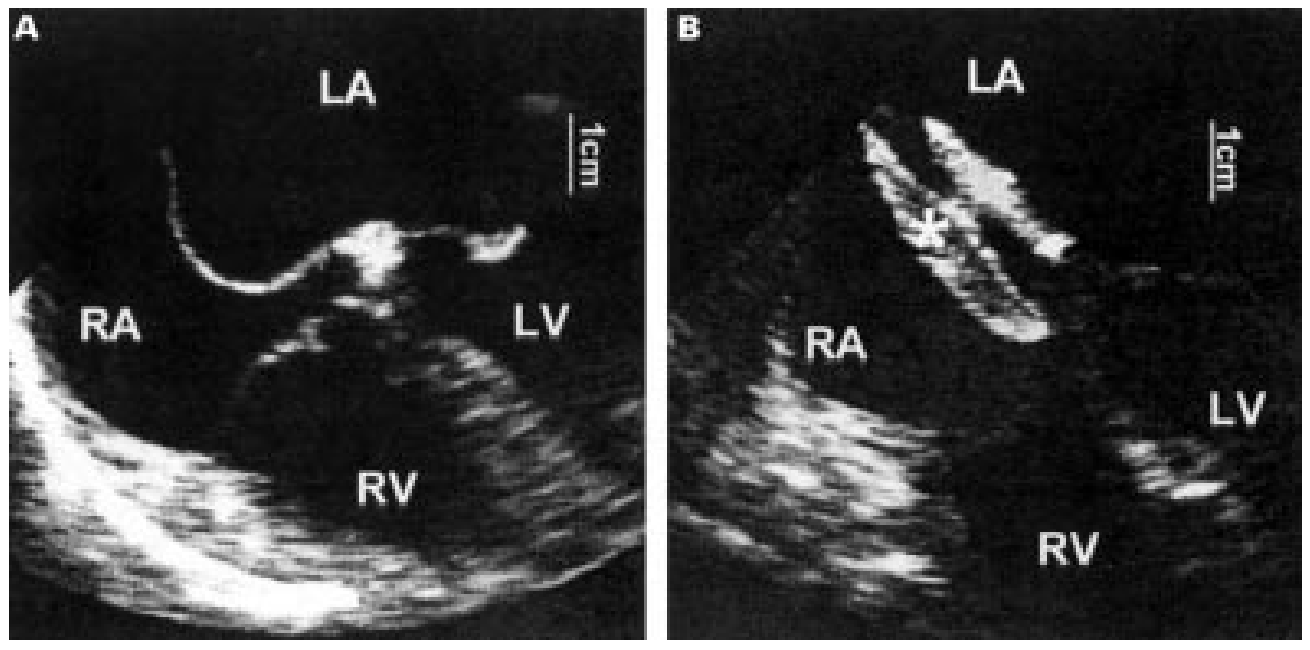

Figure 3 Transoesophageal echocardiographic four chamber view of an atrial septal aneurysm with a persistent foramen ovale before (left) and after (right) placement of an Amplatzer PFO occluder ( ${ }^{\star}$ ). The aneurysm is completely splinted. $L A$, left atrium; $L V$, left ventricle; $R A$, right atrium; $R V$, right ventricle.
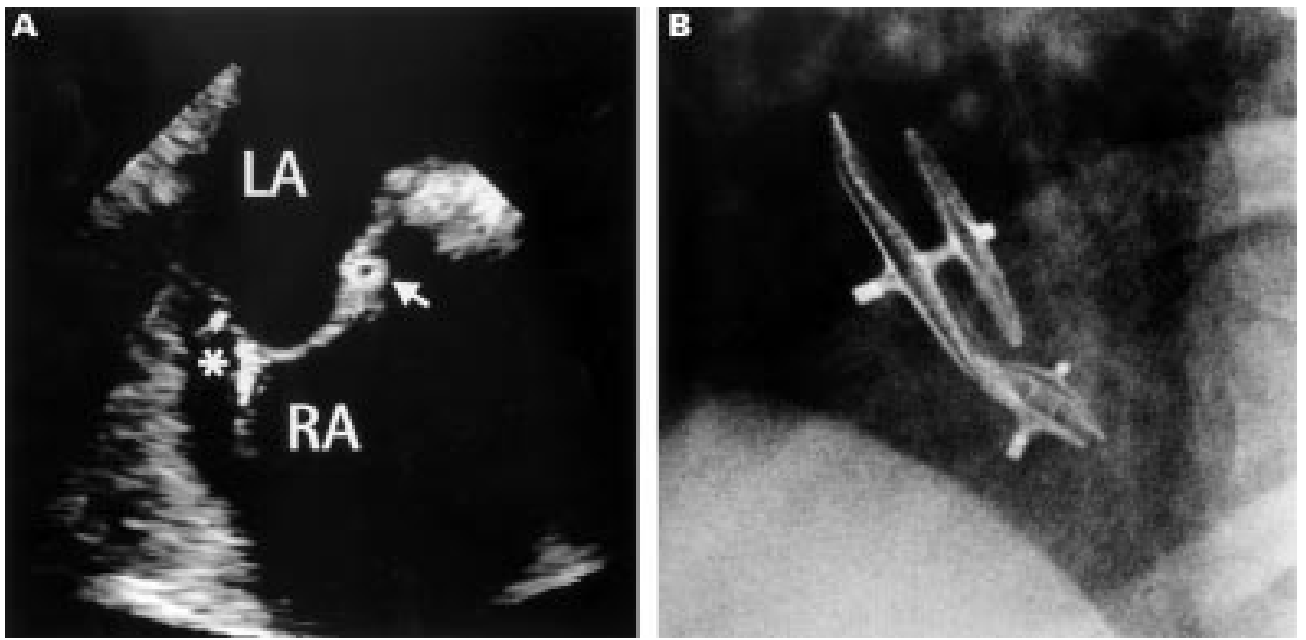

Figure 4 (A) Transoesophageal echocardiographic view of an atrial septal aneurysm with perforations in two different locations (type $C$ ). The asterix shows the site of two small perforations; the arrow points to a long sheath which is introduced across a persistent foramen ovale into the left atrium. (B) Transcatheter closure was performed with an Amplatzer PFO occluder and an Amplatzer ASD occluder (fluoroscopic view, $45^{\circ}$ left oblique and $20^{\circ}$ cranial tilt).

implantation of more than one device (type C), seven were treated with an Amplatzer and three with a Cardio-Seal device. A single device was platzer, three Cardio-Seal), with evidence of residual shunts (fig 2). One of these patients was treated with a second device (Cardio-Seal) 12 months later, and complete occlusion was d. Four others were scheduled for patients, two Amplatzer devices were placed in one session (figs 1 and 4B); no residual shunts were detected and the devices splinted the sepwell. (type D), the morphology of the perforations was thought to be unsuitable for closure by a transcatheter device (fig 5). All these patients surgery and had the perforated atrial septum was subsequently replaced with a pericardial patch. All these patients had an uneventful recovery. There were no complica- 

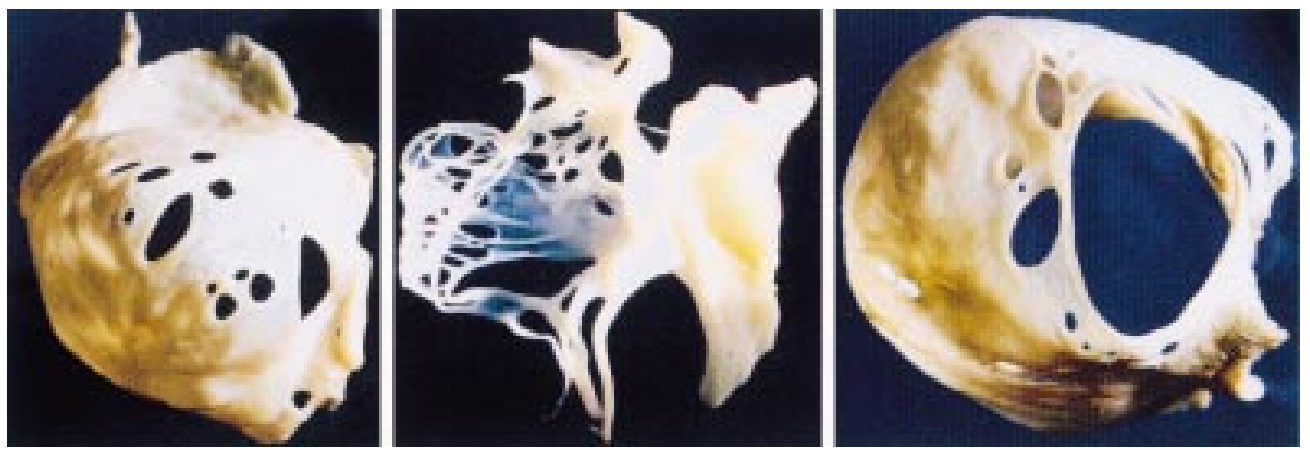

Figure 5 Specimens of surgically resected multiperforated atrial septal aneurysms (type D) which were judged as unsuitable for transcatheter closure: one aneurysm has multiple smaller perforations (left), one has network-like strands (centre), and a third has one large and multiple smaller perforations.

tions of transcatheter device placements, such as embolisation or rhythm disturbances.

During the mean follow up period of 14 (12) months (range 5-30 months), which represents 477 patient months in those treated by device placement, all devices stayed in place. In all patients treated successfully by the catheter technique after a thromboembolic event $(n=23)$, no further thromboembolic events occurred during a mean follow up period of 13 (11) months (range 5-30 months), which represents 295 patient months. In the four patients with residual shunts (fig 2), the shunts did not close spontaneously and the placement of a second device is planned.

\section{Discussion}

Our data show that perforated atrial septal aneurysms can be successfully treated by transcatheter device placement, provided the interatrial communication is not too large or too close to the mitral valve and there are no more than two perforations or small perforations in clusters of two. The latter may require the placement of two devices, while aneurysms with multiple perforations may only rarely be treatable with the currently available transcatheter devices, and may require lifelong anticoagulation or surgery. In this retrospective analysis, device closure of perforated atrial septal aneurysms was successfully accomplished in $58 \%$ of unselected cases.

\section{MORPHOLOGY OF ANEURYSM RELEVANT TO} TREATMENT

Previous investigators have used different criteria for the diagnosis of atrial septal aneurysm and have proposed different classifications. ${ }^{101314} \mathrm{On}$ the basis of the success of treatment by transcatheter device placement, we propose a clinically useful classification to facilitate the selection of symptomatic patients for device closure. The morphological features that form the basis of this classification and are relevant for successful interventional treatment are the location, number, and size of the interatrial communications. In our opinion, it is not relevant whether the aneurysm is predominantly bulging to the right or the left side of the interatrial septum, ${ }^{13}$ because we only advocate the treatment of patients with either a history of stroke and a TOE documented potential of right to left shunt through interatrial communications, ${ }^{15}$ or a significant left to right shunt that results in right ventricular volume overload, such as an atrial septal defect that is not associated with an aneurysm. While there is agreement on the need for closure of atrial septal defects with left to right shunt $(\mathrm{Qp} / \mathrm{Qs})$ greater than 1.5:1 (irrespective of the presence or absence of aneurysm), even when incidentally diagnosed in adults, ${ }^{16}$ treatment strategies for patients with perforated atrial septal aneurysm and cryptogenic stroke need to be defined. Our policy of treating patients with perforated atrial septal aneurysm and a history of transient ischaemic attacks or cryptogenic stroke may represent overtreatment,${ }^{17}$ but is also practised in other centres. ${ }^{6} 151819$ As not all of our patients with a single unexplained neurological event had documented peripheral venous thrombosis or a history of pulmonary embolism, we need a better definition of treatment criteria for the future. In our opinion, this may be achieved by prospective multicentre trials designed to establish treatment rationales based on the true prevalence of perforated atrial septal aneurysms in the general population, the rate of complications, and results of alternative medical treatment such as anticoagulation.

\section{TRANSCATHETER DEVICES USED IN THIS STUDY} Since May 1998, we have preferred to use the Amplatzer PFO occluder or the Cardio-Seal device to treat atrial septal aneurysms with small perforations, as they have a better splinting effect and may thus stabilise the aneurysm better than the Amplatzer ASD occluder. Owing to the rotation symmetry, the Amplatzer occluder is easier to implant than the Cardio-Seal device in complex aneurysms. The limbs of the Cardio-Seal occluder can be trapped through additional perforations, which complicates the delivery of a second device. However, a combination of an Amplatzer ASD occluder (larger disc on the left atrial side) and an Amplatzer PFO occluder (large disc on the right atrial side) forms a predictable and stable splint within a multiperforated aneurysm of type C (fig 4B).

Recent reports have linked the devices used in our patients to real or potential complications. Fracture of the limbs of the Cardio-Seal device has been found to occur, and there is some debate about the biological effects of 
nitinol. ${ }^{20}$ Clearly, a longer follow up is required for an accurate assessment of the true incidence of complications for these devices. When comparing the side effects from surgery and device implantation for treatment of atrial septal defects in the short term, the Amplatzer device is certainly as effective as surgery. ${ }^{21}$

\section{LIMITATIONS}

We analysed our data on atrial septal aneurysm treatment retrospectively. We have not performed a randomised study assigning patients with a history of neurological complications and perforated atrial septal aneurysms to a protocol of closure versus no closure. A substantially longer follow up period is required to study the effect of closure of perforations on the incidence of systemic thromboembolism in this group. However, the other group of patients treated with transcatheter device closure, which constituted $36 \%$ of our cases, had significant left to right shunt and required closure irrespective of the presence or absence of atrial septal aneurysm. We did not perform a formal comparison of the three different catheter delivered devices that we used, but relied on experience and clinical acumen to decide which device or combination of devices was most suitable for closure of the perforation or perforations in each individual patient.

\section{CONCLUSIONS}

Despite the limitations of this study, we conclude that our proposed classification of morphology of perforations of atrial septal aneurysm is clinically useful for selecting symptomatic patients for treatment by transcatheter devices. While there is general agreement that patients with perforated atrial septal aneurysm and associated significant left to right shunts with right ventricular volume overload should be treated, further prospective studies are required to define the need for treatment of such aneurysms in patients with a history of systemic thromboembolism.

We are grateful for editorial assistance from Tonie Derwent.

1 Agmon Y, Khandheria BK, Meissner I, et al. Frequency of atrial septal aneurysms in patients with cerebral ischemic events. Circulation 1989;20:1942-4.

2 Schneider B, Hanrath P, Vogel $\mathrm{P}$, et al. Improved morphological characterization of atrial septal aneurysm by morphological characterization of atrial septal aneurysm by transesophageal echocardiography: relation to cer
cular events. Am Coll Cardiol 1990;16:1000-9.

3 Mügge A, Daniel WG, Angermann C, et al. Atrial aneurysm in adult patients. A multicenter study using transthoracic in adult patients. A multicenter study using transthoracic and transesopha

4 Louie EK, Konstadt SN, Rao TL, et al. Transesophageal echocardiographic diagnosis of right to left shunting across the foramen ovale in adults without prior stroke. $\mathcal{F} \mathrm{Am} \mathrm{Coll}$ Cardiol 1993;21:1231-7.

5 Zabalgoitia M, Norris LP, Garcia M. Atrial septal aneurysm as a potential source of neurological ischemic events. $A m \mathcal{F}$ Card Imaging 1994;8:39-44.

6 Wos S, Bachowski R, Domaradski W, et al. Surgical treatment of atrial septal aneurysm. $\mathcal{f}$ Cardiovasc Surg 1996;37:139-42.

7 Mehan VH, Sideris EB, Meier B. Use of the reversed buttoned device to treat an atrial septal aneurysm associated with a patent foramen ovale. Br Heart $f$ 1995;74:563-5.

8 Kaulitz R, Paul T, Hausdorf G. Extending the limits of transcatheter closure defects with double umbrella device scatheter closure defects with dou
(Cardio-Seal). Heart $1998 ; 80: 54-9$.
9 Berger F, Ewert P, Stiller B, et al. Initiale klinische Erfahrungen mit dem Amplatzer Septal Occluder ein selbstzentrierendes Doppelschirmchen zum Versch

10 Hanley PC, Tajik AJ, Hynes JK, et al. Diagnosis and classification of atrial septal aneurysm by two-dimensional echocardiography: report of 80 consecutive cases. $7 \mathrm{Am}$ Coll Cardiol 1985;6:1370-82.

11 Rao PS, Langhough R, Beekmann RH, et al. Echocardiographic estimation of balloon-stretched diameter of secundum atrial septal defects from transcatheter occlusion. Am Heart f 1992;124:172-5.

12 Masura J, Gavora P, Formanek A, et al. Transcatheter closure of atrial septal defects using the new self-centering Amplatzer septal occluder: initial human experience. Cathet Cardiovasc Diagn 1997;42:388-93.

13 Olivares-Reyes A, Chan S, Lazar EJ, et al. Atrial septal aneurysm: a new classification in two hundred five adults. $\mathcal{F}$ Am Soc Echocardiogr 1997;10:644-56.

14 Roudaut R, Gosse P, Chague F, et al. Clinical and echocardiographic features of the aneurysms of the atrial septum in infants and adults; experience with 44 cases. Echocardiography 1989,6:357-62

15 Nellesen U, Daniel WG, Matheis G, et al. Impending paradoxical embolism: correct diagnosis by transesophageal echocardiography and prevention by surgery. f Am Coll Cardiol 1985;5:1002-4.

16 Konstandinides S, Geibel A, Olschewski M, et al. A comparison of surgical and medical therapy for atrial septal defect in adults. N Engl f Med 1995;333:469-73.

17 Daniel WG. Transcatheter closure of patent foramen ovale. Therapeutic overkill or elegant management for selected patients at risk? Circulation 1992,86:1013-15.

18 Marazanof $M$, Roudaut $R$, Cohen $R$, et al. Atrial septal aneurysm. Morphological characteristics in a large population: pathological associations. A French multicenter study on 259 patients investigated by transoesophageal echocardiography. Int f Cardiol 1995;52:59-65.

19 Estagnasie P, Djedaini K, Le Bourdelles G, et al. Atrial septal aneurysm plus a patent foramen ovale. A predisposing factor for paradoxical embolism and refractory hypoxemia factor for paradoxical embolism and refractory hypox

20 Rao PS, Sideris EB. Transcatheter closure of atrial septal defect [letter]. Heart 1999;82:644.

21 Berger F, Vogel M, Alexi-Meskishvili V, et al. Comparison of results and complications of surgical and amplatzer device closure of atrial septal defects $\mathcal{F}$ Thoracic Cardiovasc Surg 1999;118:674-8.

\section{CORRECTION}

Randomised double blind trial of oral versus intravenous flecainide for the cardioversion of acute atrial fibrillation. N J Alp, J A Bell, M Shahi. Heart 2000;84:37-40.

The key to figure 2 (Cumulative cardioversion rates for oral and intravenous flecainide) was printed incorrectly. The intravenous (iv) flecainide should be the open circles, and the oral flecainide the solid squares. The figure with the correct key is reproduced below.

The error is regretted.

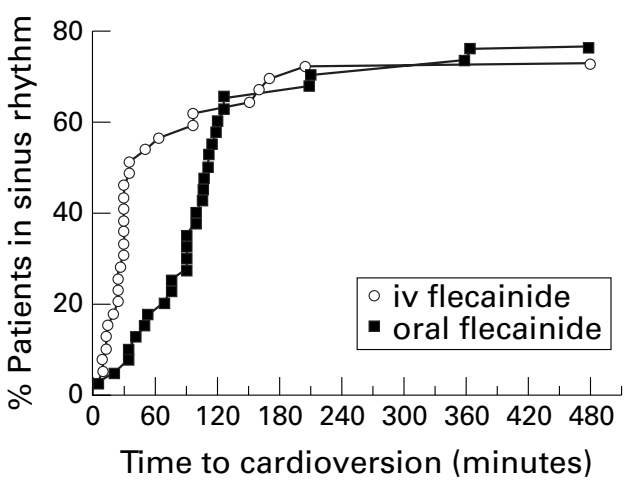

